\section{$\underset{\substack{\text { hommes } \\ \text { \& migrations }}}{ }$}

\section{Hommes \& migrations}

Revue française de référence sur les dynamiques

migratoires

\section{$1322 \mid 2018$}

Exposer les migrations

\title{
L'écomusée de Fresnes : retour sur un engagement précoce
}

Entretien avec Juliette Spire, attachée de conservation à l'écomusée du Val-de-Bièvre.

\section{Mikaël Petitjean}

\section{Q OpenEdition}

12 Journals

Édition électronique

URL : https://journals.openedition.org/hommesmigrations/6614

DOI : 10.4000/hommesmigrations.6614

ISSN : 2262-3353

Éditeur

Musée national de l'histoire de l'immigration

Édition imprimée

Date de publication : 1 juillet 2018

Pagination : $35-40$

ISBN : 978-2-919040-42-1

ISSN : $1142-852 X$

\section{Référence électronique}

Mikaël Petitjean, «L'écomusée de Fresnes : retour sur un engagement précoce », Hommes \& migrations [En ligne], 1322 | 2018, mis en ligne le 01 juillet 2020, consulté le 21 janvier 2022. URL : http://

journals.openedition.org/hommesmigrations/6614; DOI : https://doi.org/10.4000/

hommesmigrations.6614 


\section{L'ÉCOMUSÉE DE FRESNES : RETOUR SUR UN ENGAGEMENT PRÉCOCE}

Entretien avec JULIETTE SPIRE, attachée de conservation à l'écomusée du Val-de-Bièvre, réalisé par MIKAËL PETITJEAN, chargé de mission au Musée national de l'histoire de l'immigration.

\section{Hommes \& Migrations : Pourriez-vous nous rappeler brièvement l'objet de l'écomusée du Val-de-Bièvre?}

Juliette Spire : Depuis sa création en 1978, l'écomusée du Val-De-Bièvre, situé à Fresnes dans la banlieue sud de Paris, s'est fixé pour objectif d'être le reflet d'une société urbaine contemporaine, de réfléchir avec la population à son propre devenir, mais aussi de rendre compte de la mémoire collective. Françoise Wasserman, conservatrice du patrimoine et directrice de l'écomusée jusqu'à 1998, est à l'origine du projet du musée. Toute une partie de l'activité, surtout depuis 1990, consiste à travailler sur des sujets qui concernent la population fresnoise, en veillant à la faire participer de façon concrète à toutes les phases du travail de l'écomusée, de la collecte à la présentation.

\section{H\&M : L'écomusée de Fresnes est l'un des premiers acteurs à avoir présenté une exposition sur l'histoire de l'immigration. Comment cette thématique est-elle apparue au sein du musée?}

J. S. : L'écomusée s'est constitué en 1979 alors que la ruralité marquait encore le territoire et la mémoire de ses habitants. Il est pensé comme un centre culturel ayant pour mission la conservation et la mise en valeur du patrimoine naturel et culturel de l'histoire locale fresnoise. Françoise
Wasserman, conservatrice chargée du projet de l'écomusée dès sa conception, l'a teinté d'une forte attention aux « oubliés de l'histoire ${ }^{1}$ ».

La première exposition de l'écomusée en novembre 1979 porte ainsi sur L'enfant et l'école hier et aujourd'hui. En 1986, une autre exposition porte sur la thématique Blanchisseuse, laveuse, repasseuse: la femme, le linge et l'eau. Ces thématiques correspondaient aux collections de l'écomusée, plutôt orientées sur la dimension "village rural » de l'histoire de Fresnes.

Dans les années 1990, l'écomusée a pris acte de lévolution de son territoire depuis la seconde partie du XXe siècle vers une fonction de banlieue urbaine. Cette structure urbaine s'est développée à Fresnes entre le milieu des années 1950 et le milieu des années 1970, modifiant radicalement la forme du territoire, sa sociologie, son image et son mode de fonctionnement. Dès lors, ce n'est plus tant Fresnes, village francilien, mais « la banlieue » qui faisait sens. Confronté à cette situation, force a été de repositionner le territoire d'investigation de l'écomusée en direction des thématiques liées à la banlieue et à ses habitants. Cette transformation fait alors écho à l'essor des " musées de société " au début des années $1990^{2}$. Depuis 2000, ce positionnement est allé de pair avec le transfert du musée à la communauté d'agglomération du Val-de-Bièvre, dotée d'une compétence culturelle et qui élargit donc les missions du musée aux territoires de sept villes du sud de la banlieue parisienne, de Gentilly à Fresnes. 
Nous avons voulu inscrire le projet du musée dans la prise de conscience de l'urbanité, de l'appartenance à la cité et, de ce fait, participer à l'éclosion de la citoyenneté. Devenant le reflet de la société contemporaine, le musée s'est inséré dans le tissu social pour « donner à voir » à la population et lutter par-là même contre l'exclusion sociale. De ce fait, l'équipe du musée a dû se positionner en partenaire des techniciens des politiques publiques municipales, des édiles, des associations locales et communautaires, et des citoyens.

L'enjeu pour l'écomusée a donc été de proposer un projet où la dimension historique et contemporaine éclaire les problèmes de société, la marginalité, les minorités comme la citoyenneté ordinaire. Dans cette perspective, il était important de s'orienter aussi vers l'histoire de l'immigration.

\section{H\&M : De quelle façon le musée s'est-il emparé de cette thématique?}

J. S. : Dès les années 1990, lécomusée a commencé à s'intéresser à la population immigrée. Notre medium privilégié étant l'exposition, nous savions que tôt ou tard nous serions amenés à muséographier l'immigration. Avant de relever un tel défi, il nous a semblé opportun de sensibiliser le public de l'écomusée, fidèle ou non, aux problèmes de l'expression des minorités. Nous avons donc débuté par l'expression des jeunes rappeurs-taggeurs tout en renforçant notre politique d'action culturelle envers des jeunes dits de la «seconde génération ».

Des jeunes artistes ont réalisé des graffitis, notamment pour certains décors de l'exposition Hip-hop dixit sur les jeunes et le mouvement hip-hop en 1990. Son inauguration a donné lieu à un spectacle de rap associant danse et chanson. Cet axe participatif a préfiguré la programmation de l'exposition Rassemblance en 1993. Avec celle-ci, les Fresnois issus de l'immigration sont devenus informateurs, mais aussi présentateurs de leur propre patrimoine lorsqu'ils étaient sollicités pour se mettre en scène chez eux, afin d'être pris en photo.

Dans l'exposition Femmes tunisiennes, en 1997, ce sont des habitantes de Zarzis, contactées grâce à la communauté tunisienne de Fresnes, qui ont confié des objets. Lors de l'inauguration, ces femmes ont animé la soirée et préparé des mets traditionnels.

\section{H\&M : Pourriez-vous nous indiquer comment s'est montée l'exposition Hip-hop dixit, le mouv' au musée?}

J. S. : Cette initiative d'accueil de jeunes des banlieues au musée a vu le jour en 1989 sous l'impulsion du ministère de la Culture (Département des publics et de la diffusion culturelle de la Direction des musées de France) et avec la collaboration de l'association Artémisia ${ }^{3}$. Elle s'inscrivait dans une perspective globale : repenser les formes possibles d'action culturelle en direction de publics habituellement éloignés des circuits culturels classiques et donc du musée.

Le projet s'est imposé, tout d'abord, parce que le hip-hop n'est pas une mode, un phénomène éphémère, mais un état d'esprit particulièrement ancré en banlieue. Le "mouv' " comme l'appellent ses adeptes, parfois désignés par l'appellation "B Boys », est un discours : il s'agit de "s'en sortir » ou de lutter contre l'exclusion sociale, culturelle, scolaire et professionnelle avec pour «armes » des pratiques artistiques comme la danse (le break, le hype...), la musique (le rap) ou la peinture (l'art du graffiti, notamment). Le musée, à l'écoute des cultures contemporaines, devenait pour elles un lieu d'accueil, un lieu ouvert.

Une recherche ethno-historique sur les origines du mouvement hip-hop a été engagée par les B Boys de Fresnes. Elle a été suivie par des ateliers de graffiti-art, de rap et de danse qui se sont mis en place dans deux musées : l'écomusée de Fresnes et le musée Bossuet de Meaux. Divers locaux de la ville ont été mis à la disposition des jeunes pour leur permettre de progresser dans leurs disciplines. 


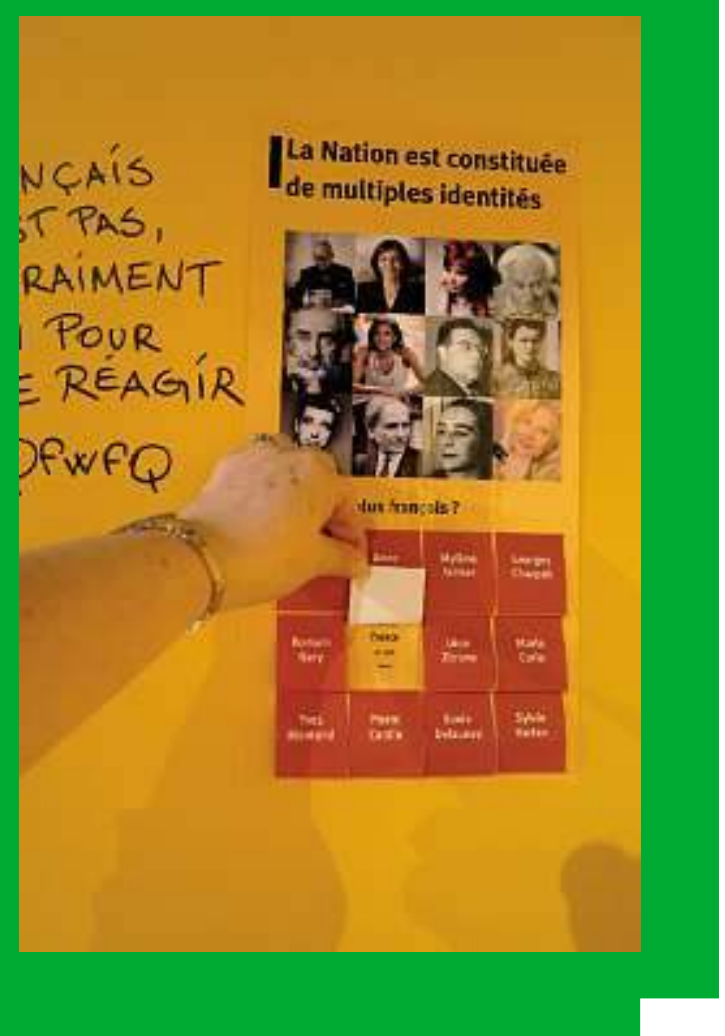

Affiche de l'exposition Paroles de femmes tunisiennes, Écomusée du Val-de-Bièvre, 1998. @ ÉcomuséE du VAL-DE-BIÈVRE, Fresnes.

l'on quitte le système scolaire. La deuxième partie présentait succinctement les différentes formes de protestation de minorités raciales ou sociales: du pacifisme de Gandhi, aux marches des Afro-Américains réprimés dans les années 1960 et à la Marche pour l'égalité et contre le racisme, dite «Marche des Beurs », en 1983, et leurs limites. La troisième partie consacrée au hip-hop présentait, à travers des photographies, des peintures et des films vidéo, les slogans pacifiques du mouvement, sa vie, ses grandes figures, le rap, la danse et les différents styles de graffitis.

De nouveaux publics se sont rendus au musée. Le jour de l'inauguration, lors de laquelle avait lieu un concert de rap, certains jeunes ont spontanément proposé de faire un «service d'ordre » et de protéger l'exposition contre d'éventuels casseurs. Les craintes de la municipalité de voir la ville se transformer et se recouvrir de graffitis n'ont pas été suivies d'effets et les jeunes sont revenus plusieurs fois à l'exposition.

\section{H\&M : Suite à ce premier projet, comment l'écomusée s'est-il lancé dans la réalisation de Rassemblance : un siècle d'immigration en Île-de-France (1992-1993) ?}

\section{H\&M : Cette recherche ethno-historique et ces ateliers ont donc nourri le contenu de l'exposition?}

J. S. : Les informations et les documents collectés par les jeunes ont servi de matière première pour l'exposition. La première partie de l'exposition s'attachait à recenser les problèmes de société que dénonce et tente de résoudre le mouvement: le racisme, le manque de reconnaissance des cultures des jeunes, l'urbanisme inhumain des grandes cités à l'origine de la formation de ghettos sociaux, la violence, les problèmes d'identité des jeunes issus de l'immigration et, enfin, la spirale de l'échec à laquelle il est difficile d'échapper lorsque
J. S. : La ville portait une dynamique de dialogue avec les communautés immigrées du territoire. Une commission extra-municipale pour la participation à la vie locale des étrangers avait été mise en place par des élus de la ville, notamment Micheline Savard, l'une des adjointes au maire. Les équipements culturels municipaux étaient encouragés à développer une politique de démocratisation culturelle. La municipalité de Fresnes avait, par ailleurs, mis en place des cours d'alphabétisation pour ces populations et marqué son soutien auprès des associations émanant de ces communautés.

Le dialogue avec les habitants d'origine immigrée était facilité par le tissu associatif. De nombreux Tunisiens faisaient partie du personnel communal 


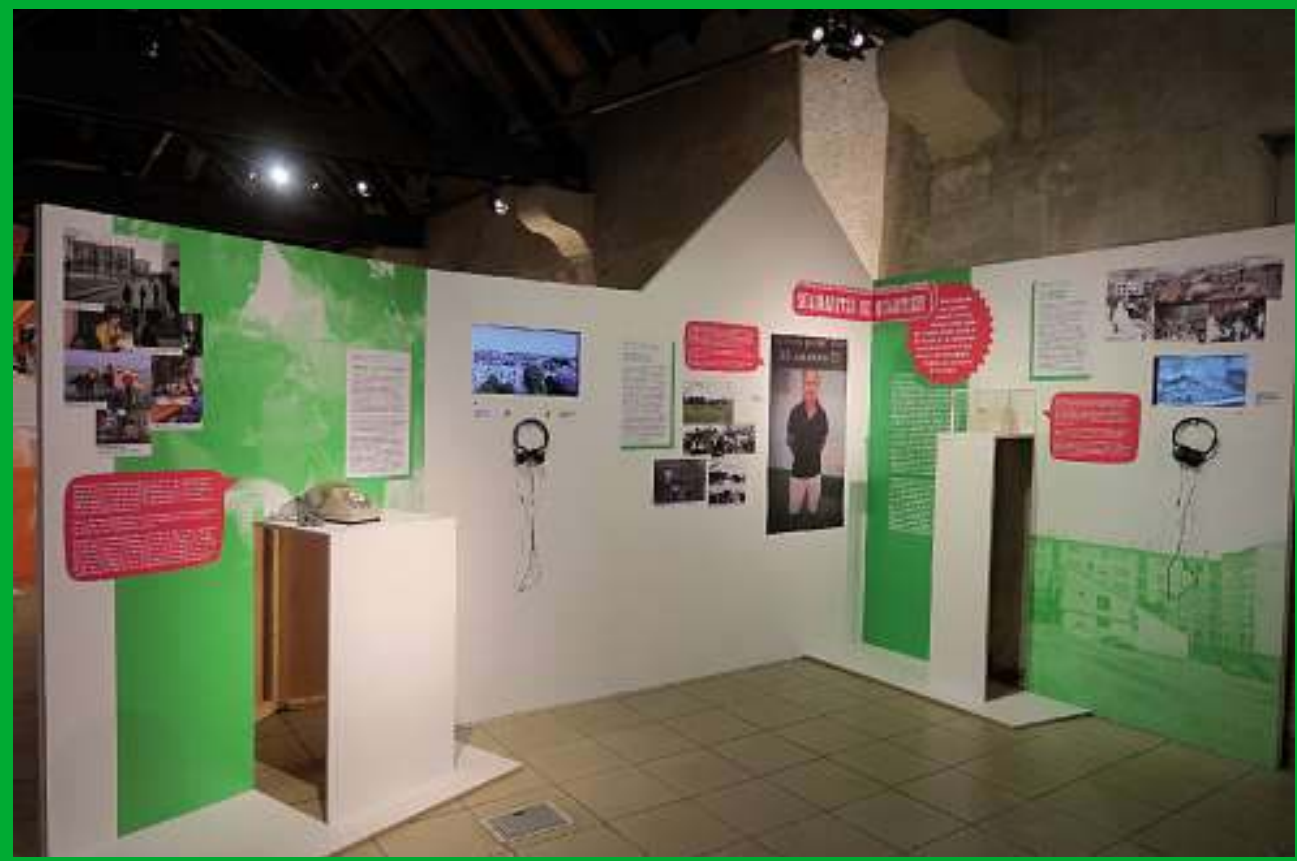

Affiche d'un appel à collecte de dons à l'écomusée du Val-de-Bièvre (1999-2000). @ ÉcomUSÉE DU VAL-DE-BIÈVRE, FrESNES.

depuis les années 1960 et animaient une Amicale des Tunisiens dynamique. Une Association de coopération et d'échanges franco-tunisienne de Fresnes (ACEFT) a par la suite été créée. Enfin, un jumelage a été institué en 1993 entre Fresnes et la ville tunisienne de Zarzis.

Une enquête avait été réalisée auprès de la population immigrée pour cerner leur appréhension de la ville et l'utilisation qu'ils faisaient des services municipaux. L'analyse des résultats nous permettait déjà de savoir quelles étaient les pratiques culturelles des immigrés dans la ville, ces derniers représentant $10 \%$ de la population globale. Il s'agissait ensuite de trouver comment dire, comment montrer, comment raconter l'immigration. Comme nous voulions donner la parole aux « communautés immigrées » et rendre compte de leurs histoires, il nous fallait trouver des interlocuteurs.

\section{H\&M : Comment s'est traduite cette approche participative ?}

J. S. : De nombreuses relations ont été nouées avec les associations d'immigrés sur l'ensemble de la région. L'écomusée a mené un travail multidisciplinaire au sein de communautés étrangères dans la ville et dans le groupement d'immeubles dit de « la Lutèce » qui comptait environ 400 habitants, dont 30 \% d'étrangers en 1990. Ce quartier pauvre rassemblait des habitants aux conditions de vie difficiles, parfois précaires, cette situation ayant tendance à empirer avec les effets de la crise qui touche plus durement des populations déjà fragilisées. L'écomusée a fait appel à l'association des locataires comme interlocuteur privilégié, ainsi qu'aux représentants des différentes communautés regroupées dans des associations culturelles. Un partenaire parisien, l'association 
$\mathrm{AIDDA}^{4}$, implantée à la Goutte-d'Or a également appuyé l'écomusée en mettant à sa disposition son fonds photographique.

La recherche a été conduite pendant plus d'une année dans le quartier de La Lutèce, principalement auprès de six familles d'origines différentes : turque, portugaise, marocaine, africaine, tunisienne et cambodgienne. Ce travail nous a permis de mieux comprendre leur vie, leur insertion dans la société, et également de constituer un fonds documentaire essentiellement photographique.

Photographies, témoignages écrits ou oraux, témoignages d'anonymes ou de plus célèbres, textes de lois constituaient la majeure partie de notre discours muséographique. Nous voulions que l'exposition fasse réfléchir, bouscule les idées reçues, facilite les dialogues futurs.

Partant du constat qu'en 1992 un Français sur cinq a un de ses grands-parents d'origine étrangère, l'exposition invitait à découvrir les « populations d'origine étrangère " arrivées depuis un siècle. Elle interrogeait les causes du départ, l'exil, les lieux de vie et de travail, le quotidien et les fêtes, la vie communautaire, l'isolement ou les regroupements ethniques et familiaux, le désir et les difficultés d'intégration.

La scénographie mettait en lumière le brassage des cultures par l'évocation symbolique de l'univers architectural du pays d'origine, des documents photographiques anciens ou récents, des témoignages, des textes littéraires ou législatifs évoquaient le contexte historique, économique et culturel qui a accompagné les vagues migratoires successives ${ }^{5}$. L'accent a été mis sur le rôle du travail et de l'appel de main-d'œuvre dans l'histoire de l'immigration.

\section{H\&M : Après cette exposition, l'écomusée a-t-il renouvelé sa programmation d'expositions temporaires ?}

J. S. : Les axes de travail de l'écomusée entre 1990 et 2000 ont été centrés sur les « minorités ». Nous étions le musée qui représentait les exclus, les " oubliés de l'histoire », et aussi des musées. Ce discours et notre orientation se sont infléchis à partir de 1999, date d'arrivée d'un nouveau conservateur à la tête de l'écomusée, Alexandre Delarge, dont l'objectif majeur est de faire évoluer le musée vers plus de participation des habitants.

Si la première exposition réalisée à cette période sur les gens du voyage en France ${ }^{6}$ se situait dans la ligne des précédentes, nous avons commencé à évoluer dans la manière d'intégrer les populations étrangères ou d'origines étrangères dans le musée au même titre que le reste de la population.

En 2000, nous avons réalisé une exposition de collecte invitant les habitants à nous apporter des objets qui témoignaient à leurs yeux du patrimoine de l'an 2000 pour les générations futures. Nous recevions les donateurs ou préteurs, et chaque objet apporté était assorti d'une petite enquête orale autour de l'usage de l'objet et de la raison de son apport au musée. Cette exposition Vos objets au musée racontent Fresnes n'a suscité que peu de visites et cela nous a questionné sur la manière de faire participer toute la population à lélaboration et à la définition de notre patrimoine commun. Cette participation était pourtant au cœur de l'affiche de l'exposition, qui figurait, entre autres, une théière pour le thé à la menthe et qui suggérait l'apport de l'immigration maghrébine dans notre patrimoine commun.

\section{H\&M : L'écomusée a-t-il fait évoluer sa méthodologie suite à cette expérience ?}

J. S. : En 2002, nous avons réalisé une exposition qui faisait suite à la collecte réalisée en l’an 2000 mais dont le processus d'élaboration a été différent. Nous sommes partis d'une question - "qu'est-ce que la banlieue? »- avec de multiples réponses possibles : un lieu multiculturel, un lieu au fort maillage associatif, un lieu vert, etc. Nous sommes allés 
trouver des habitants qui étaient concernés par les diverses thématiques. Nous les avons interrogés sur leur définition de la banlieue et, pour finir, ils devaient donner ou prêter un objet signifiant de leur discours. Notre politique de collecte a donc été basée à la fois sur l'objet, mais aussi sur la parole autour de l'usage de l'objet. Cette démarche est toujours celle que nous adoptons aujourd'hui. L'écomusée constitue ainsi une riche collection d'enquêtes orales sur l'ensemble de la population qui vit en banlieue. Il interroge, à travers sa politique d'expositions temporaires, l'histoire et l'identité du territoire dans toute sa diversité, comme en témoignent les expositions récentes En-quête d'identités (2015) et Habitants et bâtisseurs de banlieue (2017).

Notre choix est donc de faire participer davantage la population locale au travail d'élaboration et de définition du patrimoine et, ainsi, de rendre compte de l'ensemble des cultures qui composent la banlieue. Notre travail sur la parole des habitants, présent désormais de façon systématique dans chaque exposition, rend compte des trajectoires individuelles et collectives des habitants. À chaque fois, nous rencontrons les parcours de l'immigration.

Notre structure, lieu patrimonial, collecte ainsi des objets multiculturels, sans oublier la parole et les trajets qui s'y rattachent. Elle est parfois dépositaire d'archives privées liées aux relations que nous tissons avec les associations locales. Même si nous n'avons pas vocation à être un centre d'archives, nous avons ainsi reçu le dépôt des archives de
l'Association franco-tunisienne et de l'Association de jumelage avec la Roumanie. Le travail conduit autour de l'exposition Parle ma banlieue (2008) témoigne de cette méthodologie. Elle s'est accompagnée d'une collecte de paroles auprès de 160 habitants issus de sept villes de l'agglomération, notamment des quartiers populaires. Cette collecte a rendu compte de la relation des habitants à leur quartier et témoigne à la fois des plaisirs de la sociabilité en banlieue et de ses difficultés.

\section{H\&M : Cette évolution méthodologique a-t-elle conduit à redéfinir le périmètre de l'écomusée?}

J. S. : Le projet scientifique et culturel de l'écomusée du Val-de-Bièvre a évolué pour intégrer ce travail de réseaux sur le territoire afin de permettre la participation des habitants à la vie de l'écomusée. Celui-ci est devenu un centre de ressources, un lieu de compréhension du territoire qui permet la réflexion et l'action. C'est un lieu de collecte et de conservation des objets et des informations liés au territoire et à ses thématiques. Acteur du développement territorial, l'écomusée agit par le biais de démarches patrimoniales participatives pouvant être finalisées par des réalisations variées (fêtes, expositions, animations, créations...), en vue de donner la parole aux habitants. Dans ce cadre, l'objectif est toujours de permettre à l'ensemble des expressions culturelles et identitaires d'être représentées. 